Document downloaded from:

http://hdl.handle.net/10251/47911

This paper must be cited as:

Torres Cortes, A.; Tavares De Araujo Cesariny Calafate, CM.; Cano Escribá, JC.; Manzoni, P.; Ji, Y. (2015). Evaluation of flooding schemes for real-time video transmission in VANETs. Ad Hoc Networks. 24:3-20. doi:10.1016/j.adhoc.2014.07.030.

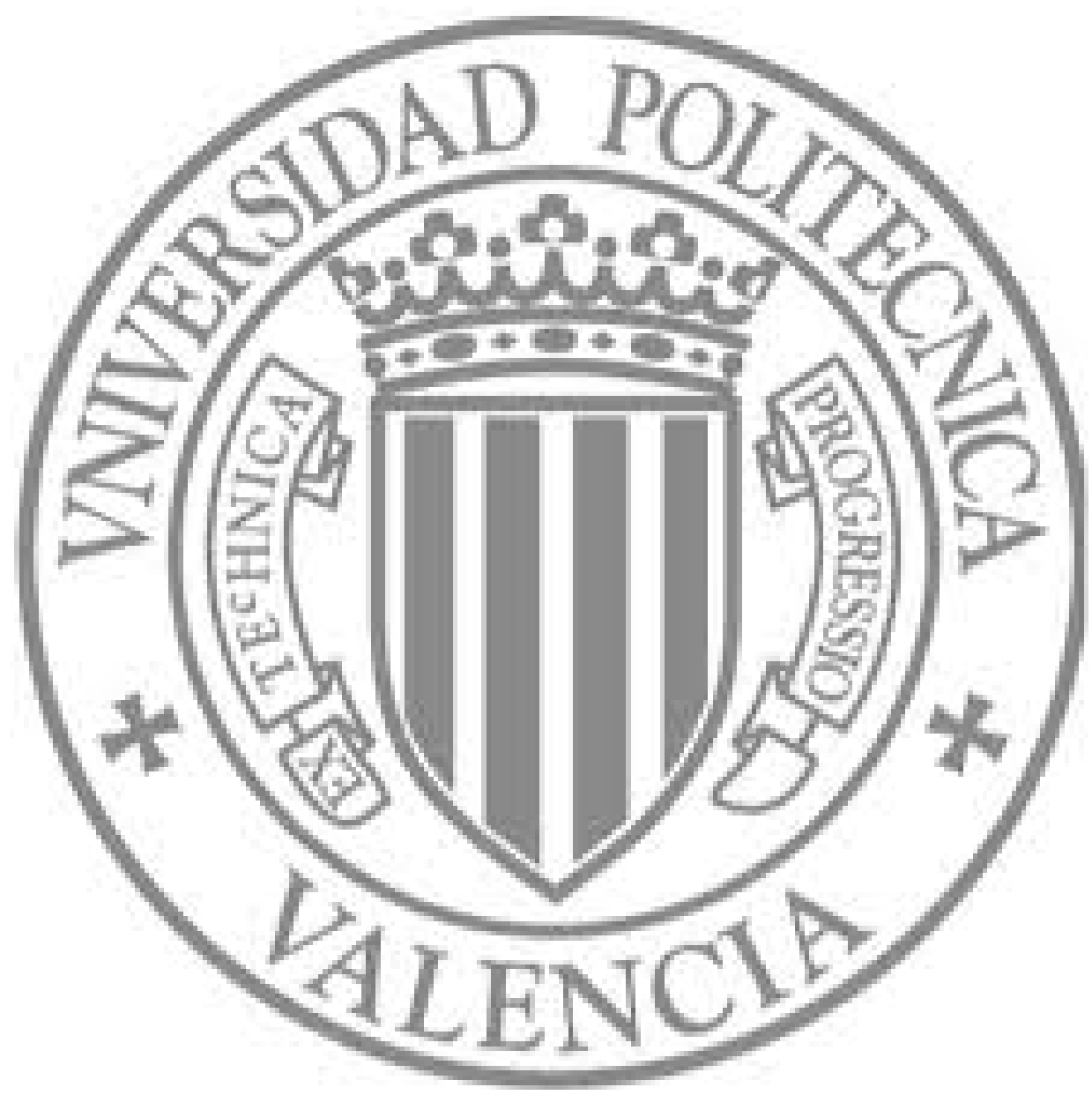

The final publication is available at

http://dx.doi.org/10.1016/j.adhoc.2014.07.030

Copyright Elsevier 


\title{
Evaluation of Flooding Schemes for Real-time Video Transmission in VANETs
}

\author{
Alvaro Torres, Yusheng Ji, Carlos T. Calafate, Juan-Carlos Cano, Pietro \\ Manzoni $^{1}$
}

\begin{abstract}
Long-distance real-time video flooding over VANETs is a challenging topic due to the dynamic nature of vehicular networks. Real-time video transmission has high requirements in terms of bandwidth and delay, while VANETs are characterized by very limited radio resources and high mobility.

Our objective is to compare ten different flooding schemes specially designed for this task, selecting the best one in terms of packet arrival ratio and PSNR. Additionally, we propose ACDB, an improved flooding scheme to cope with variable vehicle density situations.

Furthermore, to ensure a good behaviour under any type of circumstances, we also study the impact of GPS drift on these schemes. Simulation results show that long-distance real-time video flooding transmission is feasible under certain vehicle density conditions, and provides enough quality for being used in future applications.
\end{abstract}

\section{Introduction}

The research community has been interested in Vehicular Ad-Hoc Networks (VANETs) for several years since the deployment of this type of networks will be able to provide significant improvements in terms of road safety [1], as well as to obtain valuable real-time traffic information [2].

Several types of applications and protocols have been proposed to propel the possibilities that this type of network provides [3], [4]. However, every proposed application and protocol has to cope with the problems and characteristics inherent to this type of networks, such as high relative speeds, Doppler effect, low transmission rate, etc.

Automatic accident warning and notification has become a critical safety application in VANETs [5], where the majority of protocols adopted flooding techniques to warn all the nodes, as well as the traffic authorities, about the accident.

\footnotetext{
${ }^{1}$ Department of Computer Engineering Universitat Politècnica de València, Valencia, Spain atcortes@batousay.com, calafate,jucano,pmanzoni@disca.upv.es

${ }^{2}$ National Institute of Informatics, Tokyo, Japan kei@nii.ac.jp
} 
Other type of applications useful for our everyday lives relies on DTN protocols to spread traffic information, such as traffic jams, and pollution levels detected in different areas of the city [6], [7]. Both applications have in common that the amount of information to transmit can be considered "low", and both require that the information is able to reach its final destination (high reliability).

Additionally, several studies have built upon the idea of providing Video On Demand to vehicle users with a combination of V2V and V2I. Vehicles are only acting as receivers and, although video delivery implies a high amount of information, this can be scheduled and distributed among a set of RSUs, and stored in the vehicle's buffer.

The recent approval of the new H.265 video compression standard [8], which intends to replace the widely used and well-known H.264 standard [9], provides a new opportunity for real-time video transmission in critical contexts. The new standard, which outperforms the old one achieving the same video quality with only $50 \%$ of the bit-rate [10], is expected to become an enabling technology when attempting to provide real-time video transmission in vehicular networks; thus, H.265 was selected to carry out the different experiments in the scope of our work.

The motivation of this paper is to address the challenges that arise when attempting to provide an innovative service in vehicular environments: vehicles involved in traffic accidents shall produce a high amount of information (a video sequence), which is of interest to all the nodes in the network (both vehicles and RSUs), and this information flow must reach its destination within a low delay (soft real-time).

This work does not attempt to present an application that makes use of this type of traffic, focusing instead on evaluating the effectiveness of different flooding schemes with the purpose of achieving a long-distance real-time video transmission under different circumstances, such as different vehicle densities and different degrees of GPS accuracy.

The objective of this work is to study the possibility of achieving live video streaming between vehicles at long distances.

The rest of the paper is structured as follows. In section 2 we review the state of the art in terms of both flooding in wireless networks and video transmission over VANETs. Afterwards, in section 3, all the different flooding schemes are described. In section 4 we provide the details of the simulation scenario, as well

as an overview of the methodology adopted. Section 5 presents the obtained results, and finally, in section 6 , we summarize the conclusions obtained.

\section{Related work}

The IEEE 802.11p protocol is the standard for Vehicle to Vehicle (V2V) communications, and it is based upon the IEEE 802.11 protocol and its quality of service extensions, IEEE 802.11e. So, IEEE 802.11 p provides a contentionbased broadcast mechanism, and its behaviour is closely related to IEEE 802.11; 
therefore, different solutions proposed for the original standard can be adapted to this new type of networks.

During the past few years, several authors have proposed different algorithms to achieve efficient flooding in MANET and VANET environments, proposing several ways of controlling the broadcast storm problem.

Yu-Chee Tseng et al. [11] presented some basic algorithms to solve this problem. The same authors published [12] an improved version of the same algorithms by adding adaptive conditions to further reduce the broadcast storm problem. In particular they proposed and improved versions of the CounterBased, Distance-Based, and Location-Based schemes. These schemes have some weak points, such as failing to provide any kind of delivery guarantee. Instead, they have some interesting features including (i) low overhead, (ii) being highly adaptable to several conditions, and (iii) providing a completely autonomous broadcast system.

Martínez et al. [13], following the guidelines of the Distance-Based scheme, proposed a flooding mechanism that takes into account the specificities of VANETs; in particular, they tweak it to provide a fast dissemination of accident alerts in urban scenarios by using information such as town layout to achieve a smarter flooding. For highway scenarios the algorithm does not significantly differ from the Distance-Based approach. Also, although a really high warning rate is achieved, they do not guarantee the alert delivery to every node in the network.

Other authors, like Osafune et al. [14], introduce the concept of "Backfire" for flooding schemes. This concept proposes that, when a node receives a copy of a message from another node which is considered to be a better re-transmitter, the first node cancels the retransmission of the packet, thereby achieving reductions in terms of sent messages and channel contention.

In order to increase both efficiency and message delivery ratio, Wu et al. [15] present a sender-oriented flooding scheme called FUZZBR, which proposes a mechanism of next-hop selection based on fuzzy logic. In order to make a good node selection they gather information by using a beaconing system, and then classifying the neighbours according to the distance, degree of mobility and RSSI of the received messages. FUZZBR also tries to guarantee message delivery using a timeout ack-based mechanism, so if a selected node does not rebroadcast the sent message, the source node will retry the sending.

Ros et al. [16] try to ensure the message delivery by addressing the problem of temporary disconnections which occur in VANET scenarios.. They make use of the beaconing system to piggyback acknowledgements in beacon messages, allowing nodes to start the retransmission of a message when some neighbour has not received the alert message.

With the same idea of making use of the beaconing system to ensure a proper message delivery through acknowledgment piggybacking, Na Nakorn et al. [17] present DECA. This flooding scheme requires a modified beaconing system with a variable beacon interval time to achieve a good behaviour. Additionally, the flooding algorithm is only based on 1-hop neighbour density information, thereby avoiding the use of GPS. Authors claim that GPS precision and availability is critical for GPS-based flooding schemes. 
To avoid the GPS availability problem, typically ocurring in tunnels, Yan et al. [3] propose a grid-based on-road localization system. According to their results, the positioning error can be higher than $10 \mathrm{~m}$. Additionally, they update the node position every two seconds and so, during that interval, the node position can be considered "old". In fact, even vehicles with GPS receivers take some time to update their position, which can lead to high deviations from the real position. Thus, it becomes critical to evaluate the performance of GPSbased flooding algorithms under these circumstances.

Despite video transmission over VANETs is a topic addressed in some recent articles, most authors propose video transmission as an entertainment technology alone, within the scope of "infotainment". In these situations the video is streamed from Road Side Units (RSU) to vehicles. On the contrary, this work focuses on distributing live video streaming between vehicles, and from vehicles to some distant RSU. Meng Guo et al. [4] presented several scenarios where this scheme is both feasible an desirable.

F. Soldo et al. [18] presented the SUV protocol, a distributed solution to disseminate video streams in VANETs. The protocol proposes dividing the neighbours into four sectors, and selecting as a candidate for rebroadcasting one node in every sector, although a special MAC layer is required to support TDMA scheduling; such requirement prevents its implementation on actual IEEE $802.11 p$ devices. Additionally, it requires very precise clock synchronization, which should be achieved using GPS. Nevertheless, as mentioned before, we assume that nodes can lose GPS signals, which is prone to cause clock synchronization problems.

Overall, although the aforementioned works provide a good number of statistics, none of them presents actual video quality results such as PSNR, being unaware of decoding problems that typically arise, such as the interdependence between frames.

A first approach towards a proper simulation environment capable of representing real-time video transmission in VANETs was proposed in [19]. Authors present a simulation platform and PSNR results, following the guidelines presented in [20], thereby providing a useful approach on how to accurately simulate video transmission.

With respect to previous works, in this paper we present a performance evaluation of different real-time video flooding schemes, evaluating the error resilience in terms of PSNR for the new H.265 video coding standard. Also, we show the robustness of the different flooding schemes when the positioning system is not precise.

\section{Description of the flooding schemes}

In this section we will briefly describe the behaviour of the different flooding

algorithms implemented. Figure 1 shows the different requirements in terms of GPS or Beaconing system of each scheme, as well as the original scheme each one is based upon. 


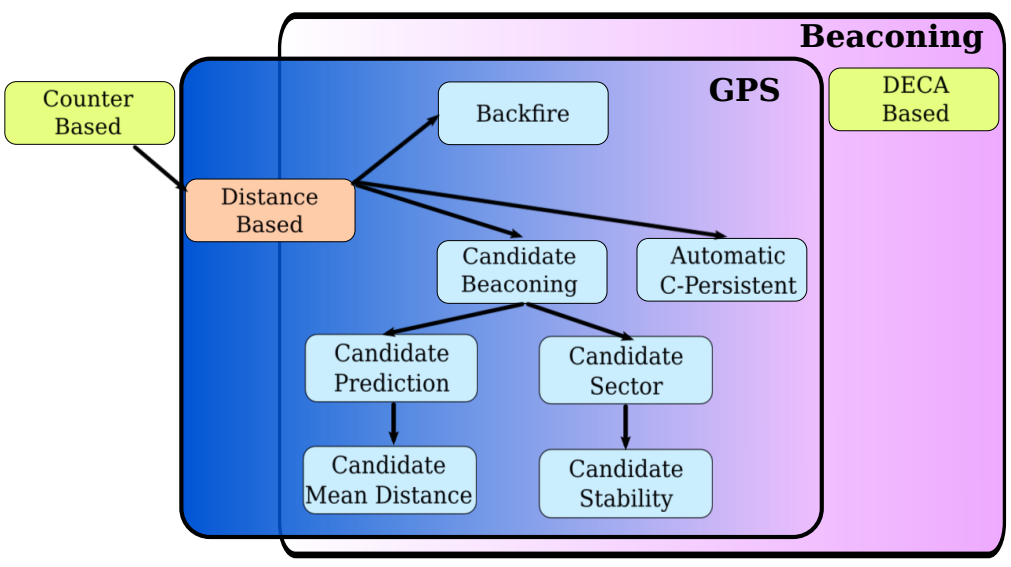

Figure 1: Inheritance diagram of the different flooding schemes.

As shown, all the different algorithms require GPS, except the DECA-Based and Counter-Based ones, whereas the beaconing system is required by every algorithm except the Counter-Based, the Distance-Counter-Based, and Backfire schemes.

\subsection{Basic schemes}

\subsubsection{Counter Based Scheme}

This scheme was one of the first schemes proposed to effectively reduce the broadcast storm problem. The main idea is that every node resends a packet until it has received $C$ copies of that packet from other nodes.

To avoid a large number of collisions when receiving a packet, every node waits from a random time before resending it, so it can listen to the medium and wait for the $C$ counter to increase.

All the flooding schemes presented in this paper, except DECA, will adopt the main idea of receiving $C$ copies of a packet to stop rebroadcasting it.

This scheme has several advantages over other solutions. The first one is that it is quite easy to implement, not having any relevant requirements except for a wireless card. The second advantage is the possibility of tuning the algorithm by modifying both the maximum amount of time a node can wait to rebroadcast a packet, and the number of copies a node should hear to stop rebroadcasting; this way, merely by modifying a simple parameter, we are able to increase the redundancy. The third advantage is that it is a good reference for other algorithms and, in case of problems, with any of the required modules (GPS or Beaconing system), it can be used as a fall-back scheme without dramatically degrading the flooding performance.

The reason for selecting the Counter-Based scheme instead of other more sophisticated schemes, such as [15], [16] and [17], is the type of traffic we intend to transmit (video traffic), especially when fast spreading is more relevant than high reliability. 


\subsubsection{Distance Based Scheme}

Based on the Counter Based Scheme, this scheme adds a new requirement: a positioning system (GPS).

Due to this new requirement, we can easily improve the intelligence of the algorithm to decide which node can be a better next-hop when rebroadcasting a packet.

Since the algorithm should remain autonomous, and since the decision about whether to rebroadcast a message should be taken independently by each node, this scheme makes use of the waiting time to resend messages. The time a node waits for before resending is inversely proportional to the minimum distance between the original node and the resending node. This way, nodes are virtually ordered according to the additional area of coverage, prioritizing those nodes whose additional area is supposed to be bigger.

To implement this scheme we need some kind of location mechanism, such as GPS, and the packets should be marked with the original sender node position and the resending node position.

This mechanism allows a rapid spreading of the packets while obtaining a profitable message forwarding process in terms of extra coverage area achieved by every new transmission.

\subsection{Adapted schemes}

\subsubsection{DECA-Based}

DECA-Bewa [17] is a flooding scheme that does not rely on positioning mechanisms to spread information. Instead, it makes use of the beaconing system to estimate the 1-hop neighbour density and, thus, select as resending node the neighbour with more 1-hop neighbours. It also makes use of the beaconing mechanism to piggyback acknowledgement messages.DECA-Bewa was adapted to video streaming requirements by including in beacons information about the last packet properly received. With this information nodes can trigger the resend of the rest of the packets not received by a certain node.

\subsubsection{Backfire Scheme}

This algorithm shares a common basis with the Distance Based Scheme but now, as proposed in [14], if it receives a packet from a node that is supposed to provide more additional coverage area, it stops rebroadcasting that packet. This strategy allows reducing the number of collisions by minimizing the number of transmissions.

\subsection{Proposed schemes}

\subsubsection{Candidate Beaconing}

If we make use of the other resource (Beaconing system) we can obtain pretty valuable information, such as the position of 1-hop neighbours.

As stated previously, the furthest neighbour is the one that is going to provide more additional coverage area, making packet rebroadcasting more beneficial. 
Source-based flooding schemes usually propose a node to rebroadcast the packet. They wait for a certain amount of time to hear the rebroadcast (which serves also as an ACK mechanism), and, in case of failure, the sender node resends the message. The main problem of these schemes is having a lot of waiting periods, which makes them unfeasible for real-time video transmission.

Instead, we introduce the concept of "Candidate". Every time a vehicle sends a packet it selects one or more of its neighbours, and proposes them as candidates. These candidates should rebroadcast the packets immediately, while the rest of the vehicles should wait for a minimum amount of time before rebroadcast the packet. The main difference towards pure source-based algorithms is that, in this case, enforcing a minimum waiting time allows the receiver node to face a less congested medium. So after this minimum waiting time they behave the same than the Distance Based scheme.

In this proposed scheme, and as a first approach, the candidate selection is done simply by selecting the furthest node in our neighbour list.

\subsubsection{Candidate Prediction}

Usually the beaconing system does not have an updated information about every neighbour position since position information is only updated when a new beacon is received, which occurs once every few seconds, and so it can easily lead to position deviations higher than $50 \mathrm{~m}$.

If every vehicle adds information about its speed and direction to beacons, we can easily estimate the current position of each of our neighbours, and thereby discard the ones that are further away than the furthest beacon received.

This strategy allows selecting a better candidate than merely using old positioning information.

\subsubsection{Candidate Mean Distance}

According to the Nakagami Fading Channel model and standard signal propagation theory, the furthest a node is, the more difficult it is for that node to properly receive a packet.

In order to improve the candidate selection, and based on the predicted positioning information, the Candidate Mean Distance scheme selects a candidate with a high probability of receiving the packet, but far enough to guarantee that the additional area coverage is large enough. So, in this case, it selects the closest neighbour for a distance of at least $60 \%$ of the distance to the furthest beacon received.

\subsubsection{Candidate Sector}

In order to improve the critical problem of selecting the best candidate, if the positioning system can provide compass information, the list of neighbours can be divided into several sectors according to their relative position.

As an example, in a highway scenario a set of two sectors can be defined (front and back). If a packet is received from a node in its "front" sector, it should select the candidate from the "back" sector to improve the coverage area. 
This mechanism can be easily extended to cities by adding more sectors, such as, front, back, left and right. So, if a packet is received from the "right" sector, we can select up to three candidates, one for each of the other sectors.

This space division attempts to avoid the spreading of messages back to a position close to the sender node. Previous works also introduce this mechanism, but they require MAC-layer modifications [18].

In addition, since this scheme is based on the Candidate Beaconing Scheme, so the candidate for each sector is the furthest node.

\subsubsection{Candidate Stability}

One of the key factors of a good candidate selection is the quality of a certain neighbour as a relay node.

Some previous works [15] rely on individualized RSSI information. In a real world scenario, that information cannot be obtained through a standard UDP socket, having to rely instead on the goodness of the driver implementation of each manufacturer, or instead on external solutions, such as adding a second wireless interface in monitor mode.

In order to achieve a highly-compatible and easy to implement solution, we propose measuring the reliability of each of our neighbours by using the beaconing system. Every node should inform, in every sent beacon, the estimated time for the next one, allowing the receivers of that beacon to estimate the amount of lost beacons.

This scheme proposes as candidate the furthest node with the lowest amount of lost beacons. In addition, since it is based on the previous Candidate Sector scheme, so in every sector it selects the most stable candidate for rebroadcasting.

\subsubsection{Automatic Copies Distance Based (ACDB)}

The ACDB scheme makes use of the density information provided by the beaconing system, as well as the number of queued packets, to dynamically adjust the value of $C$ and the maximum waiting time avaliable to rebroadcast a packet.

With this scheme we intend to avoid the candidate selection present in the sender-based schemes, and we try to provide better support for high density and low density environments while maintaining at least the same behaviour than the other schemes.

The behaviour of this scheme is to reduce the value of $C$ and to increase the Waiting time when the density is high, and to increase $C$ and reduce the Waiting time when the density is low. The first approach attempts to reduce the number of rebroadcasts and, thereby, the number of collissions, while the second one arrempts to increase the redundancy.

The value of $C$ can range from 1 to 5 , meaning that the node will stop rebroadcasting after receiving $C$ extra copies of the message. Concerning the Waiting Time, it can range from $100 \mathrm{~ms}$ to $500 \mathrm{~ms}$. The values of parameters $C$ and Waiting Time are coupled into five levels of density, being the values $C=1$, Waiting Time $=500 \mathrm{~ms}, C=2$, Waiting Time $=400 \mathrm{~ms}$, and so on. 
Table 1: Vehicle types and associated probability.

\begin{tabular}{|c|c|c|c|}
\hline Vehicle Type & Maximum Speed (m/s) & Length $(\mathrm{m})$ & Probability \\
\hline \hline Truck & 25 & 12 & 0.10 \\
\hline Car & 33 & 4 & 0.79 \\
\hline Slow Car & 25 & 5 & 0.10 \\
\hline Fast Car & 39 & 4 & 0.01 \\
\hline
\end{tabular}

The local density is estimated by making use of the number of 1-hop neighbours reported by the beaconing system. Every node clasifies the current number of neighbours into five levels, each one corresponding to certain values of $C$ and Waiting Time, as defined above. The clasification is made in a linear way between 1 and the maximum number of 1-hop neighbours ever detected.

\section{Scenario and Methodology.}

The simulation environment is composed by three main components:

- OMNeT $++[21]$, an event-driven simulator which provides a base for implementing several types of models.

- INET framework [22], an implementation of the different network models for the $\mathrm{OMNeT}++$ simulator, which includes several models from the physical to the application layer.

- SUMO (Simulation of Urban MObility) [23], which provides realistic vehicle behaviour. SUMO runs coupled with the OMNeT ++ simulator by using TRaCI, thereby allowing several mobility parameters, such as vehicle speed, to be changed in simulation time.

The transmission range in the INET framework is not defined as a fixed distance. Instead, it requires tuning different parameters such as the frequency or the level of attenuation with distance. To achieve the highest degree of similarity with reality we adopted the parameters proposed by Báguena et al. [24].

For a more realistic mobility behaviour that includes vehicle overtaking we defined a set including different vehicle types with an associated probability of occurrence. All this data can be seen in table 1 .

To perform our experiments with predefined vehicle densities we used VACaMobil [25], a tool that allows defining a vehicle arrival rate, maintaining a stable mean number of vehicles throughout the entire simulation.

Additionally, the SUMO step value was configured to $0.1 s$ in order to achieve a more realistic scenario, as when vehicles move at highway speeds $(30 \mathrm{~m} / \mathrm{s})$ the default SUMO step (1s) provides a level of granularity that is too coarse for our experiments. 


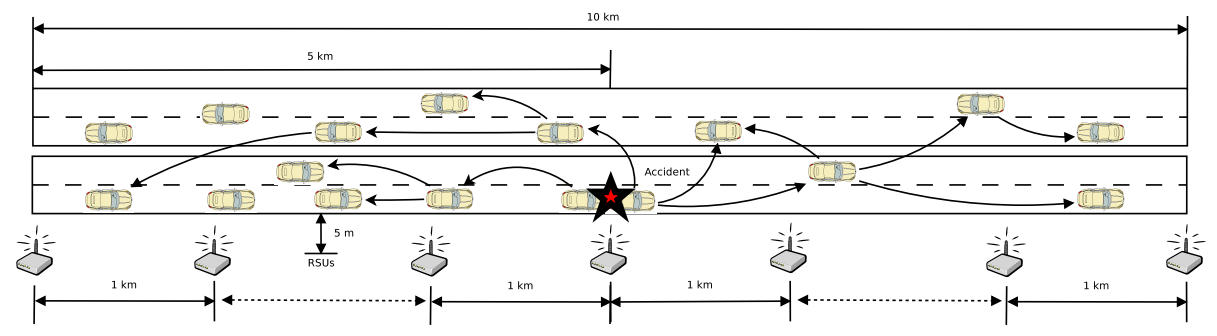

Figure 2: Highway scenario.

\subsection{Scenario}

Figure 2 shows the simulated scenario, which is a 10 kilometer straight highway with two ways and two lanes per way.

A set of RSUs has been deployed at every kilometer, and the distance between the RSUs and the road is $5 m$. RSUs do not cooperate in the flooding mechanism, being mere traffic sinks used to measure the expected quality at every kilometric point of the highway.

For our simulations we varied the vehicle arrival rates to compare the effectiveness of the protocols at different vehicle densities.

When the number of vehicles in the network becomes stable we schedule an accident at the center of the scenario ( $5 \mathrm{~km}$ point). The accident produces a small trafic jam in kilometers 0 to 5 . Futhermore, when the schedulled vehicle experiences the accident, it starts the video transmission and disseminates it through flooding.

\subsection{Methodology}

To evaluate the 10 different algorithms under the same traffic conditions, we run a set of 25 repetitions per configuration with different node mobility patterns, thereby achieving a good data significance.

We first compare the effectiveness of the different flooding schemes in terms of packet arrival ratio and video PSNR. Then, we study the impact of the positioning accuracy on the different flooding schemes.

Packet arrival ratio is shown for every scheme, while for PSNR results we selected four representative schemes.

The selected schemes are ACDB, Distance Based, Candidate Stability and Backfire. The Distance Based scheme is selected as a reference algorithm. Candidate Stability is selected because is the best performing scheme among the source-based schemes. Finally, Backfire and ACDB are selected because they are the best performing algorithms in some of the cases.

\subsubsection{Video quality measurement}

The actual transmitted video is the CIF version of the "Highway" video sequence, which contains 2000 frames [26]. The video has been encoded using the H.265 reference codec, obtaining a quality level of $37.87 \mathrm{~dB}$. This quality 


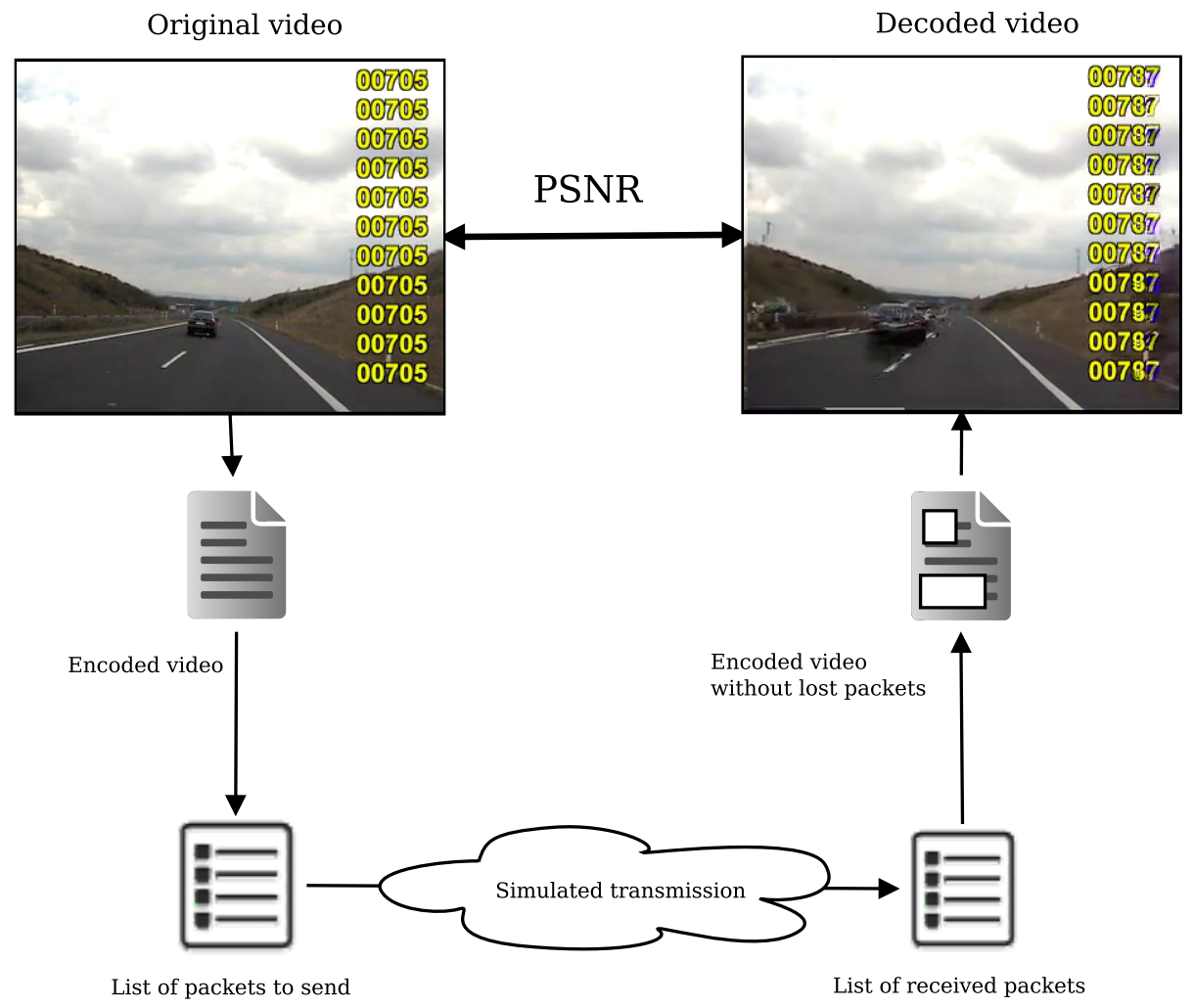

Figure 3: Overview of the video quality measurement.

level corresponds to a data rate of $283 \mathrm{kbit} / \mathrm{s}$. The encoder was configured to create RTP packetized streams. To avoid large dependencies between frames, and to try to achieve a better packet loss resilience, key-frames are generated every two seconds.

Additionally, to add real-time constraints, we assume a video buffer of $1 s$, meaning that a vehicle will start the video playback $1 s$ after the arrival of the first packet, and discarding every packet received beyond this jitter threshold.

Figure 3 shows the process followed to obtain the PSNR.

The first step is to encode the original video using the H.265 video codec, and extracting the list of packets to be sent. Every packet is a tuple which contains the transmission time, a packet id, and the size of that packet $(<$ time, id, size $>$ ).

After simulating the transmission, we delete from the encoded video sequence those packets that have not been received, thus obtaining a file of the encoded video with some gaps. That file is decoded to obtain a degraded video sequence. For decoding we employed a modified version of the reference decoder (HM 9.0) presented by Piñol et al. [27], which is able to successfully decode H.265 sequences in the presence of losses. 


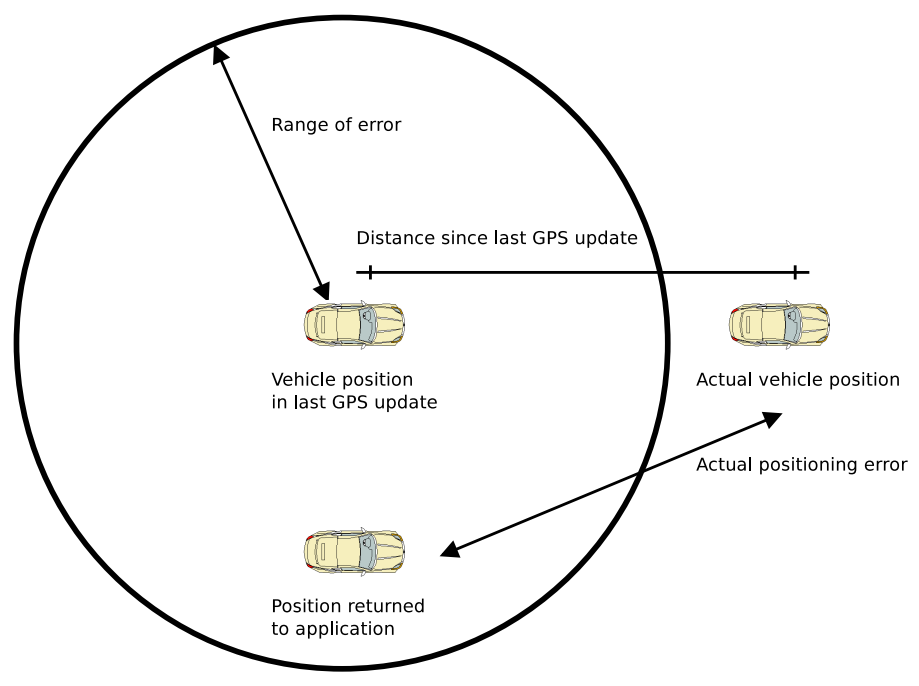

Figure 4: GPS error emulation.

The degraded video obtained is compared against the original video to obtain the PSNR.

\subsubsection{GPS emulation}

In order to achieve a realistic scenario, two different types of errors have been introduced.

The first one is GPS precision, that is, when the vehicle receives a new position it has a certain error, and so the emulated position is set within the range of the real position.

The second one is the GPS update interval. Real GPS devices update the position periodically (typically $1 s$ ). To emulate this behaviour, the application level has only access to the estimated position of the last GPS update.

Figure 4 shows the difference that exists between the actual vehicle position and the position received at the application level, evidencing the error introduced.

\subsubsection{Simulation setup}

The different flooding schemes have been tested using three vehicle density values. These densities are achieved by setting an exponential Inter-Arrival Time of vehicles equal to $0.25 s, 1 s$, and $3 s$, respectivelly. These Inter-Arrival Times correspond, approximately, to a mean density of 100,30 , and 10 vehicles $/ \mathrm{km}$, which have been labeled as "High", "Medium", and "Low" densities in the figures of this paper.

Aditionally, for every single algorithm and vehicular density setup, we tested all the possible combinations between $C$ and Waiting Time. Possible $C$ values are 1,2,3,4, and 5; Waiting Time values can be of 100,200,300,400, and 500ms. 
Table 2: Parameters for best-case simulations without positioning error.

\begin{tabular}{|c|c|c|c|c|c|c|}
\hline Density & \multicolumn{2}{|c|}{ High } & \multicolumn{2}{c|}{ Medium } & \multicolumn{2}{c|}{ Low } \\
\hline & C & Time $(\mathrm{ms})$ & C & Time $(\mathrm{ms})$ & C & Time $(\mathrm{ms})$ \\
\hline \hline Backfire & 2 & 300 & 3 & 200 & 5 & 300 \\
\hline Distance Based & 1 & 300 & 2 & 500 & 2 & 500 \\
\hline Candidate Stability & 1 & 500 & 1 & 300 & 3 & 300 \\
\hline
\end{tabular}

Table 3: Parameters for best-case simulations with positioning error.

\begin{tabular}{|c|c|c|c|c|c|c|}
\hline & \multicolumn{2}{|c|}{ High } & \multicolumn{2}{|c|}{ Medium } & \multicolumn{2}{c|}{ Low } \\
\hline & C & Time $(\mathrm{ms})$ & C & Time $(\mathrm{ms})$ & C & Time $(\mathrm{ms})$ \\
\hline \hline Backfire & 2 & 300 & 3 & 500 & 3 & 200 \\
\hline Distance Based & 1 & 300 & 1 & 300 & 3 & 500 \\
\hline Candidate Stability & 1 & 500 & 2 & 300 & 3 & 300 \\
\hline
\end{tabular}

The different results presented correspond to the best possible configuration of every algorithm, for each density, in terms of parameters $C$ (Number of copies to stop resend) and Waiting Time (Maximum time that a node waits to resend a message). The optimal parameters for the selected schemes are shown in tables 2 and 3.

In the case of the proposed flooding scheme (ACDB), none of the parameters is fixed, being their value chosen in real time by every node based on local density information.

\section{Results}

In this section we will present experimental results, obtained using the simulation setup and methodology described above.

\subsection{Comparison of flooding schemes}

The top part of figure 5 shows the percentage of received packets for all the different flooding schemes under high vehicle density, while the bottom part shows the PSNR obtained for the selected flooding schemes. We can appreciate that most schemes are able to provide more than $92 \%$ of packet arrival ratio at a distance of $5 \mathrm{~km}$ from the accident. We can also notice that ACDB is able to improve the packet arrival ratio between kilometers 1 and 4 . The Backfire scheme performs better than any other at the a distance of $5 \mathrm{~km}$. This is due to the reduced number of collissions produced when using this scheme. On the contrary, if we focus on the performance between kilometers 5 to 10 we can notice that the Backfire scheme performs slightly worse than from kilometers 0 to 5 ; this is due to a lower vehicle density.

Overall we can see that the DECA-Based scheme is the worst among the presented algorithms. Due to the high requirements of video traffic in terms of bandwidth, a high number of collisions occur when vehicles piggyback the 
information of the received packets. Notice that if vehicles that lost a packet schedulle a high number of retransmissions, eventually, that collapses the wireless medium.

In terms of PSNR, the differences between the selected algorithms are very noticeable. ACDB achieves more than $34 d B$ for a distance up to $4 k m$, while the rest of the algorithms are not able to maintain this level of quality. All the proposed schemes, except DECA, are able to maintain the quality level above $30 d B$, a value which we consider as the lower bound for a proper video visualization.

Figure 6 shows the results for the medium density scenario. In this scenario the ACDB flooding scheme is able to provide the best results up to $4 \mathrm{~km}$ achieving a delivery ratio of more than a $90 \%$. If we focus on the Candidate approach we can see that the Candidate Stability scheme is able to perform slightly better than the rest, evidencing the importance of a proper selection of the candidate to resend the packet.

With this density, the behaviour of the DECA-based scheme improves but is still highly compromised.

Results for the low density scenario are shown in figure 7. All flooding schemes evaluated in this article is unable to provide a good percentage of received packets when the density of vehicles is low. If we focus in the PSNR results we can see that the received video is unable to achieve $30 d B$ at any distance.

\subsection{Impact of GPS drift}

As seen in section 2, some authors state that the errors in the positioning sytem can affect the reliability of the different GPS-based algorithms.

For this purpose, we tested a feasible case of positioning error. The difference between the real position and the GPS stated position is in a range of $10 m$, and the frecuency of the position updates is set to $2 s$. This configuration can lead, in a worst case scenario to a deviation of about $85 \mathrm{~m}$.

Figure 8 shows the results for the high density scenario. Notice that all flooding schemes remain mostly unnafected by the positioning error thanks to the high vehicle density, which is able to compensate for this error.

Figure 9 shows the percentage of received packets for the medium density scenario. In this scenario the different schemes lose a bit of efficiency. The ACDB scheme is still able to maintain a video quality above $30 d B$ at a distance of $3 \mathrm{~km}$.

In general terms, the results show that the implemented flooding schemes do not require a precise GPS.

As stated in the previous case, for the low density scenario shown in figure 10, all the different flooding schemes are unable to achieve a percentage of received packets higher than a $90 \%$.

We can notice that the Backfire scheme suffers from a severe degradation in terms of packet arrival ratio. All other flooding schemes are able to maintain similar packet arrival ratios, with only minor differences among them. 

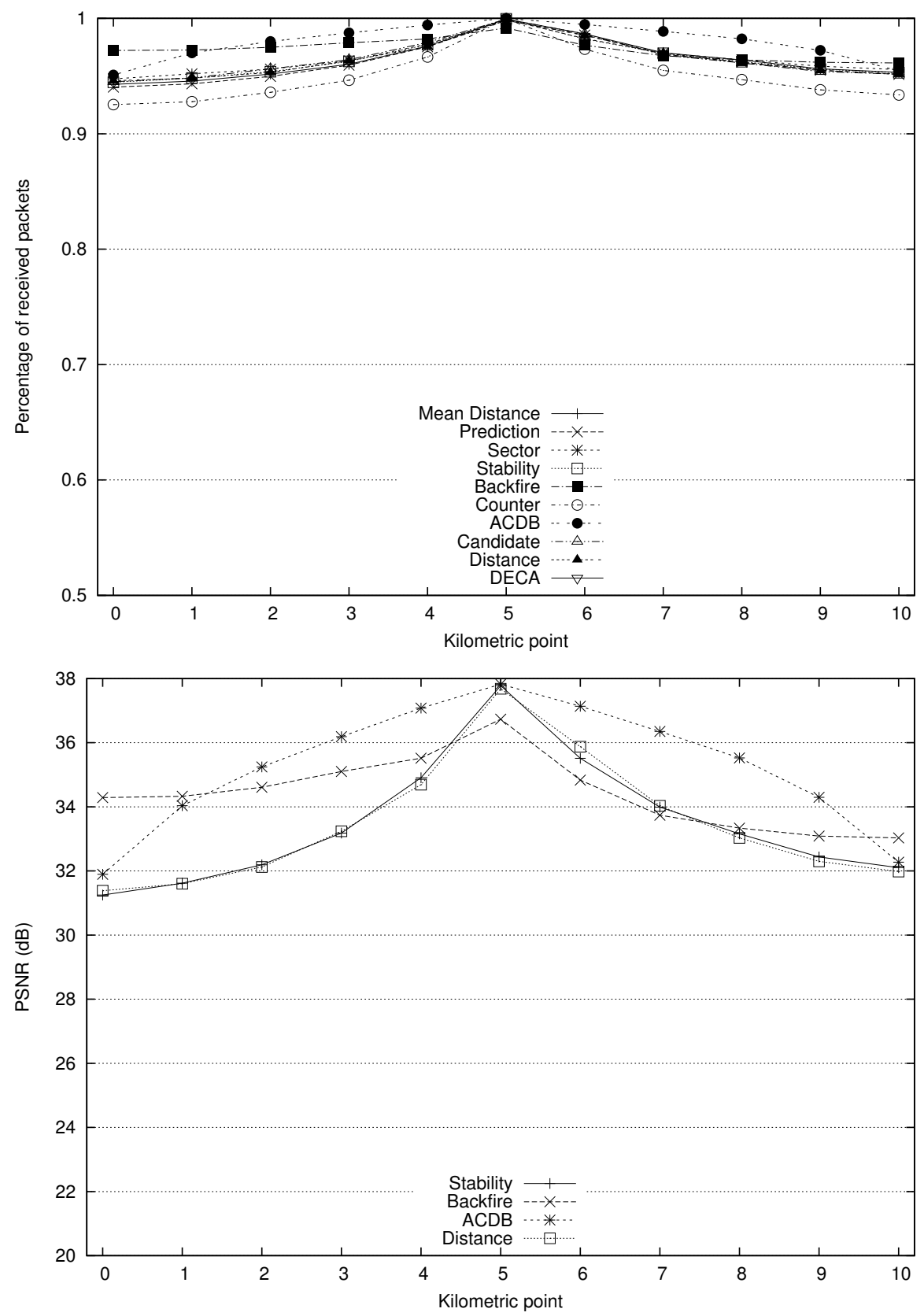

Figure 5: High density scenario. Percentage of packets received (up) and PSNR (down). 

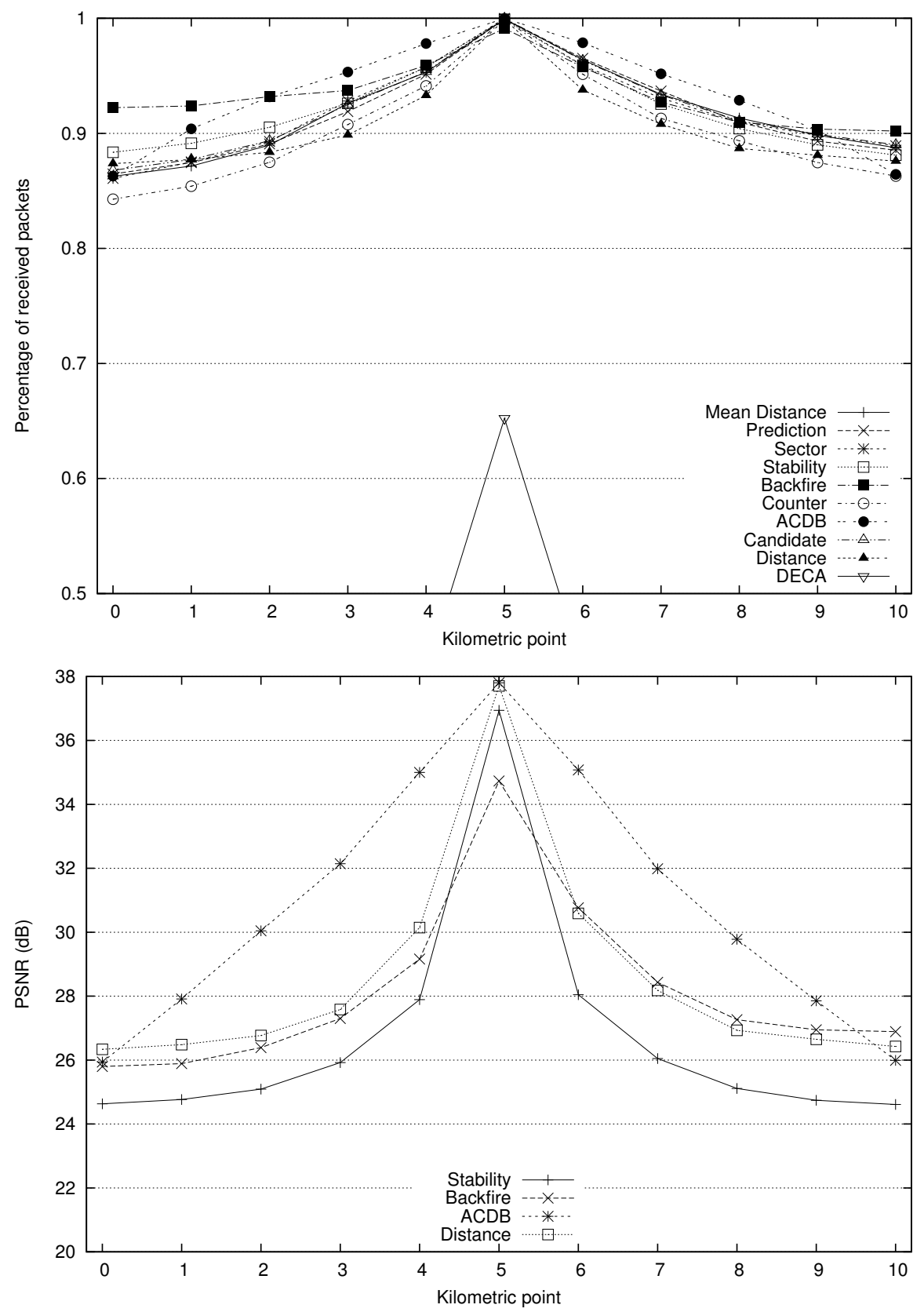

Figure 6: Medium density scenario. Percentage of packets received (up) and PSNR (down). 

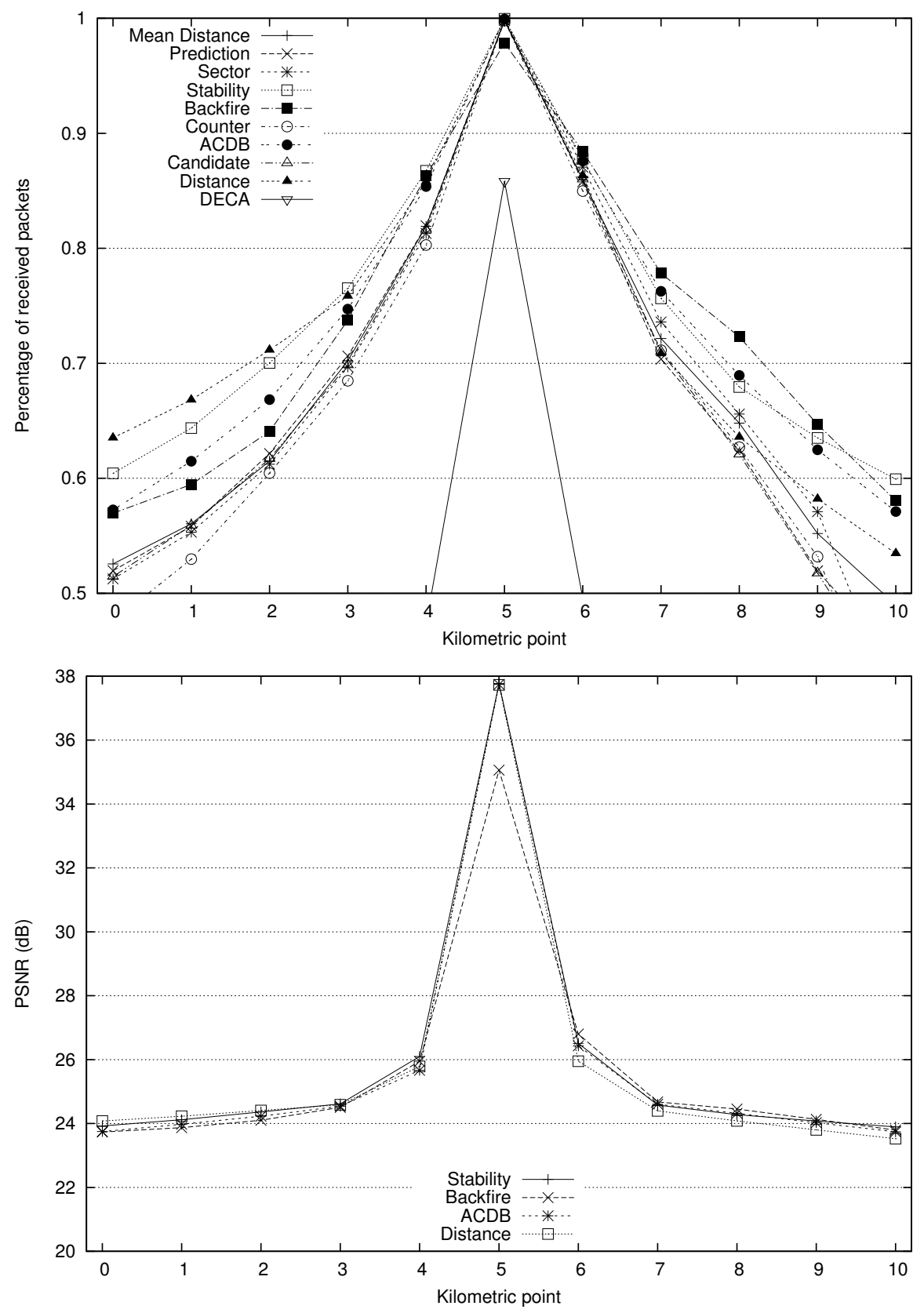

Figure 7: Low density scenario. Percentage of packets received (up) and PSNR (down). 

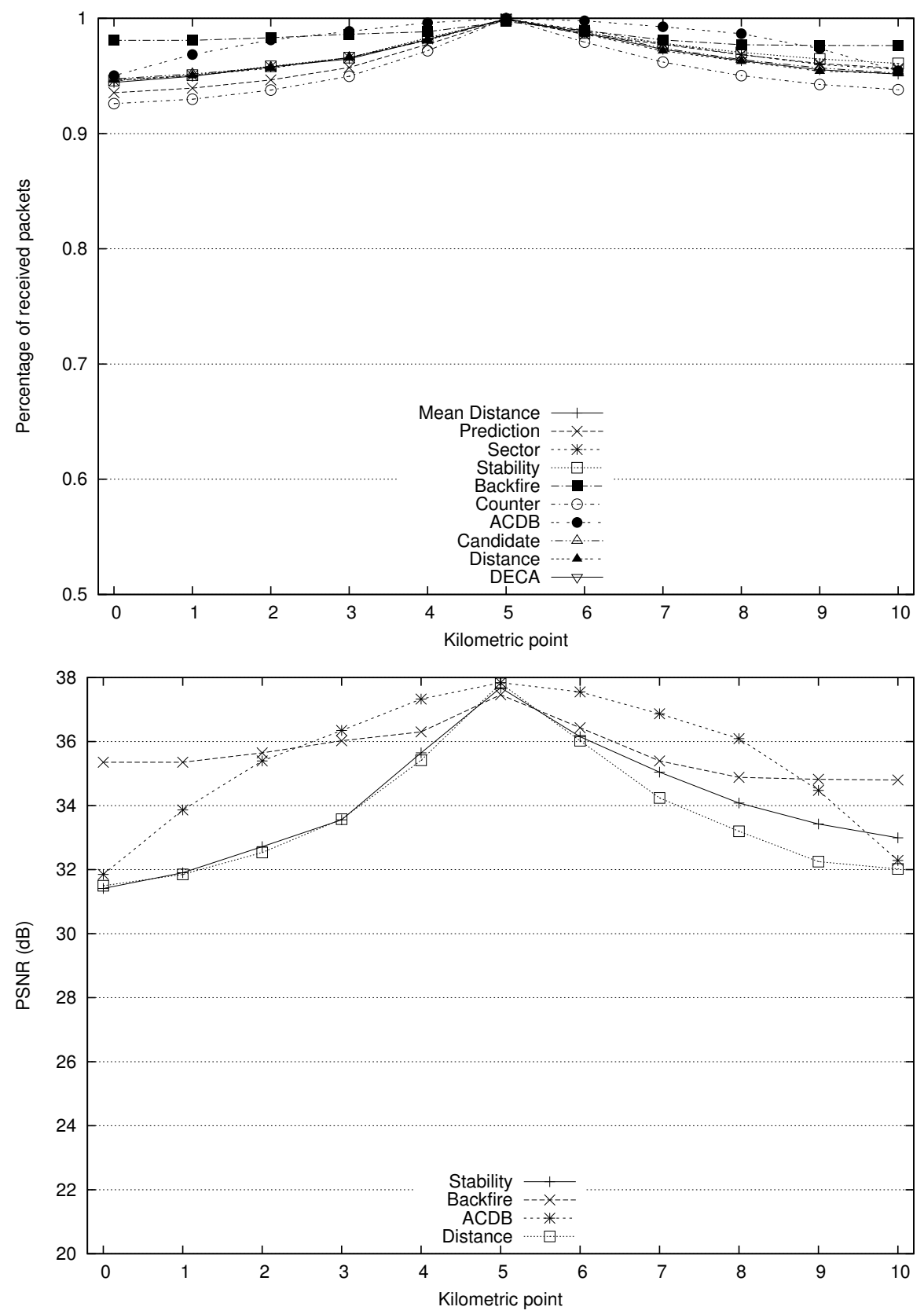

Figure 8: High density scenario. Percentage of packets received (up) and PSNR (down). 

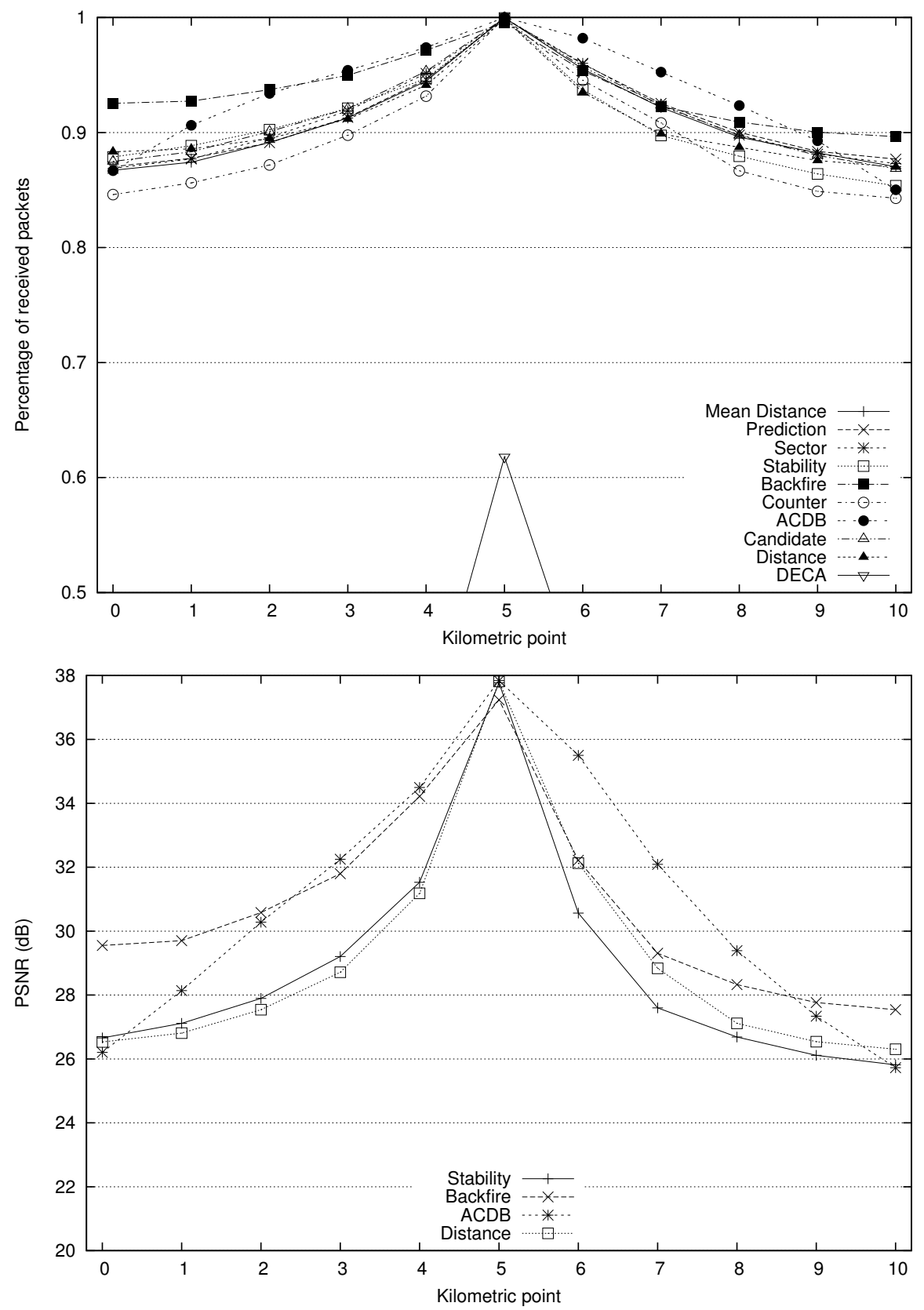

Figure 9: Mediumdensity scenario. Percentage of packets received (up) and PSNR (down). 

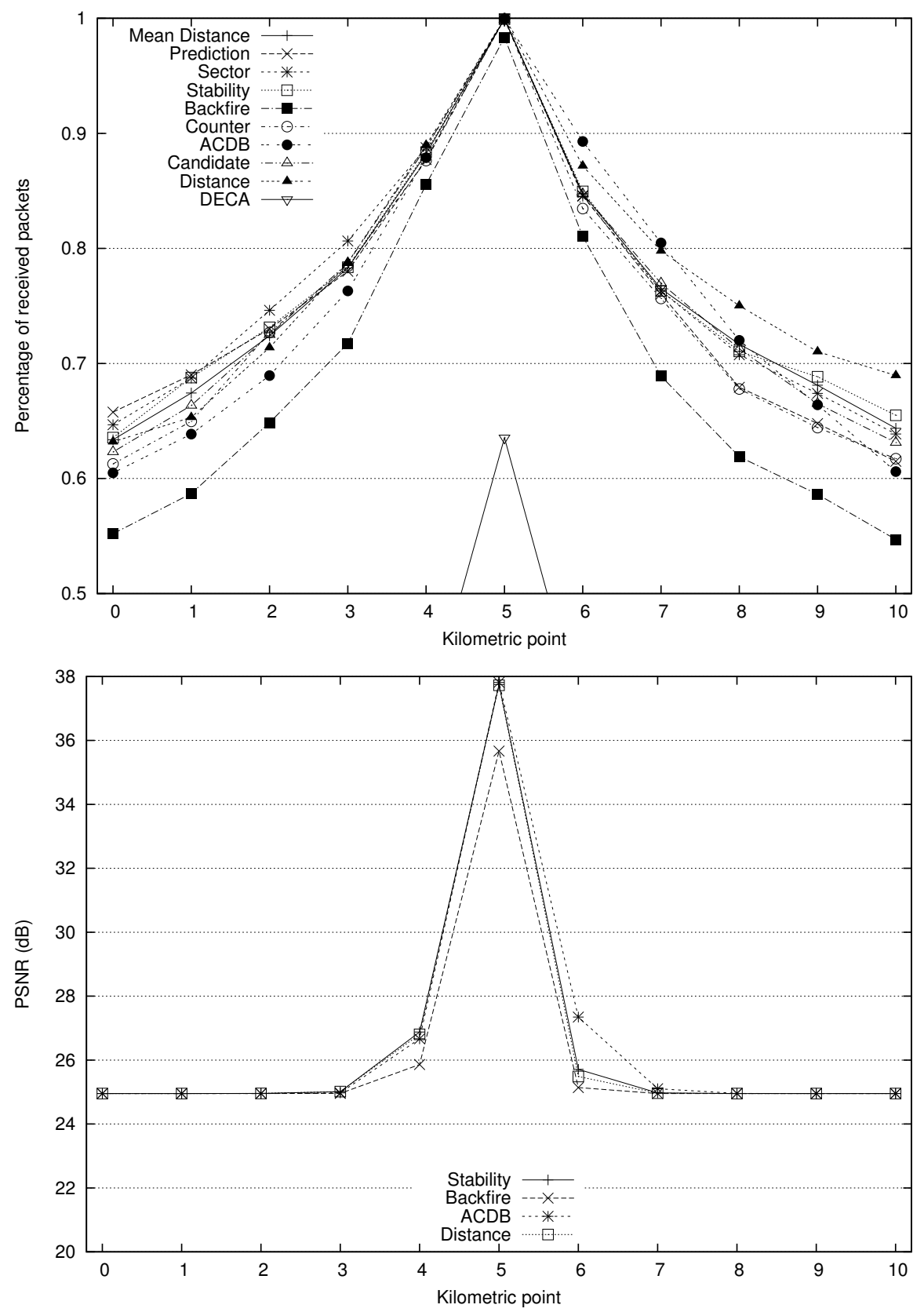

Figure 10: Lowdensity scenario. Percentage of packets received (up) and PSNR (down). 
Despite achieving packet arrival ratios higher than $60 \%$, the different flooding schemes are unable to provide a good quality in terms of PSNR, being all the results for the schemes below the $30 \mathrm{~dB}$ mark even at a distance of just $1 \mathrm{~km}$.

Overall, we find that vehicle density is a key factor to enable real time video flooding in VANETs. Also, by taking into account the behaviour of DECA, we find that flooding schemes that intend to increase the reliability by using a piggyback system are unsuitable when the traffic load is high. Also, we find that the proposed ACDB scheme is able to perform properly without any special requirements in terms of configuration and parameter tunning.

\section{Conclusions}

In this paper we analized 10 different flooding schemes for real time video transmission in vehicular environments, providing descriptive metrics such as, packet arrival ratio and PSNR. Aditionally, we evaluated the different schemes under a severe positioning error evidencing that the presented schemes are not significantly influenced by the accuracy of positioning data.

Results show that vehicle density is a key factor when attempting to achieve live video streaming. In fact, under low densities of vehicles, video streaming under acceptable conditions is not possible even at low distances. With a medium density of vehicles, live video streaming has an acceptable video quality up to $4 \mathrm{~km}$, even with imprecise positioning data. With a high vehicle density a good video quality can be achieved for distances up to $5 \mathrm{~km}$.

The Automatic Counter Distance Based (ACDB) flooding scheme is able to obtain a high percentage of delivered packets within low delay bounds without any special configuration requirement, and is able (in most of the cases) to outperform the majority of the flooding schemes when optimally configured.

\section{Acknowledgements}

This work was partially supported by the Ministerio de Economía y Competitividad, Spain, under Grant TIN2011-27543-C03-01, by the Ministerio de Educación, Spain, under the FPU program, AP2009-2415, and by the National Institute of Informatics International Internship Program.

[1] M. Fogue, P. Garrido, F. Martinez, J. Cano, C. Calafate, P. Manzoni, A system for automatic notification and severity estimation of automotive accidents, Mobile Computing, IEEE Transactions on PP (99) (2013) 1-1. doi:10.1109/TMC.2013.35.

[2] J. A. Sanguesa, M. Fogue, P. Garrido, F. J. Martinez, J.-C. Cano, C. T. Calafate, P. Manzoni, An infrastructureless approach to estimate vehicular density in urban environments, Sensors 13 (2) (2013) 2399-2418. doi:10.3390/s130202399.

URL http://www.mdpi . com/1424-8220/13/2/2399 
[3] T. Yan, W. Zhang, G. Wang, A grid-based on-road localization system in vanet with linear error propagation (2014). doi:10.1109/TWC.2013.122313.130547.

[4] M. Guo, M. Ammar, E. Zegura, V3: a vehicle-to-vehicle live video streaming architecture, in: Pervasive Computing and Communications, 2005. PerCom 2005. Third IEEE International Conference on, 2005, pp. 171-180. doi:10.1109/PERCOM.2005.53.

[5] F. Martinez, C.-K. Toh, J.-C. Cano, C. Calafate, P. Manzoni, A street broadcast reduction scheme (sbr) to mitigate the broadcast storm problem in vanets, Wireless Personal Communications 56 (3) (2011) 559-572. doi:10.1007/s11277-010-9989-4. URL http://dx.doi.org/10.1007/s11277-010-9989-4

[6] S. M. Tornell, C. T. Calafate, J.-C. Cano, P. Manzoni, Assessing the Effectiveness of DTN Techniques Under Realistic Urban Environments, 2013.

[7] K. Mershad, H. Artail, M. Gerla, We can deliver messages to far vehicles, Intelligent Transportation Systems, IEEE Transactions on 13 (3) (2012) 1099-1115. doi:10.1109/TITS.2012.2183124.

[8] H.265 standard, http://www.itu.int/rec/T-REC-H.265.

[9] H.264 standard, http://www.itu.int/rec/T-REC-H.264.

[10] H. Koumaras, M. Kourtis, D. Martakos, Benchmarking the encoding efficiency of h.265/hevc and h.264/avc, in: Future Network Mobile Summit (FutureNetw), 2012, 2012, pp. 1-7.

[11] S.-Y. Ni, Y.-C. Tseng, Y.-S. Chen, J.-P. Sheu, The broadcast storm problem in a mobile ad hoc network, in: Proceedings of the 5th Annual ACM/IEEE International Conference on Mobile Computing and Networking, MobiCom '99, ACM, New York, NY, USA, 1999, pp. 151-162. doi: $10.1145 / 313451.313525$.

URL http://doi.acm.org/10.1145/313451.313525

[12] Y.-C. Tseng, S.-Y. Ni, E.-Y. Shih, Adaptive approaches to relieving broadcast storms in a wireless multihop mobile ad hoc network, Computers, IEEE Transactions on 52 (5) (2003) 545 - 557. doi:10.1109/TC.2003.1197122.

[13] F. Martinez, M. Fogue, M. Coll, J.-C. Cano, C. Calafate, P. Manzoni, Evaluating the impact of a novel warning message dissemination scheme for vanets using real city maps, in: M. Crovella, L. Feeney, D. Rubenstein, S. Raghavan (Eds.), NETWORKING 2010, Vol. 6091 of Lecture Notes in Computer Science, Springer Berlin Heidelberg, 2010, pp. 265-276. doi:10.1007/978-3-642-12963-6-21. 
[14] T. Osafune, L. Lin, M. Lenardi, Multi-hop vehicular broadcast (mhvb), in: ITS Telecommunications Proceedings, 2006 6th International Conference on, 2006, pp. 757-760. doi:10.1109/ITST.2006.289011.

[15] C. Wu, S. Ohzahata, T. Kato, Vanet broadcast protocol based on fuzzy logic and lightweight retransmission mechanism., IEICE Transactions 95-B (2) (2012) 415-425.

URL http://dblp.uni-trier.de/db/journals/ieicet/ieicet95b.html\#WuOK12

[16] F. Ros, P. Ruiz, I. Stojmenovic, Reliable and efficient broadcasting in vehicular ad hoc networks, in: Vehicular Technology Conference, 2009. VTC Spring 2009. IEEE 69th, 2009, pp. 1-5. doi:10.1109/VETECS.2009.5073834.

[17] K. N. Nakorn, K. Rojviboonchai, Deca-bewa: Density-aware reliable broadcasting protocol in vanets, IEICE Transactions 96-B (5) (2013) 1112-1121.

[18] F. Soldo, C. Casetti, C. Chiasserini, P. Chaparro, Video streaming distribution in vanets, Parallel and Distributed Systems, IEEE Transactions on 22 (7) (2011) 1085-1091. doi:10.1109/TPDS.2010.173.

[19] P. Piñol, O. López, M. Martínez, J. Oliver, M. P. Malumbres, Modeling video streaming over vanets, in: Proceedings of the 7th ACM workshop on Performance monitoring and measurement of heterogeneous wireless and wired networks, PM2HW2N '12, ACM, New York, NY, USA, 2012, pp. 7-14. doi:10.1145/2387191.2387194.

URL http://doi.acm.org/10.1145/2387191.2387194

[20] P. Seeling, M. Reisslein, Video transport evaluation with h.264 video traces, Communications Surveys Tutorials, IEEE 14 (4) (2012) 1142-1165. doi:10.1109/SURV.2011.082911.00067.

[21] OMNeT++ simulator, http://www.omnetpp.org/, acessed: April 4, 2013.

[22] INET framework, http://inet.omnetpp.org/, acessed: April 4, 2013.

[23] M. Behrisch, L. Bieker, J. Erdmann, D. Krajzewicz, Sumo - simulation of urban mobility: An overview, in: SIMUL 2011, The Third International Conference on Advances in System Simulation, Barcelona, Spain, 2011, pp. 63-68.

[24] M. Baguena, C. Calafate, J. Cano, P. Manzoni, Towards realistic vehicular network simulation models, in: Wireless Days (WD), 2012 IFIP, 2012, pp. 1-3. doi:10.1109/WD.2012.6402805.

[25] M. Baguena, S. Tornell, A. Torres, C. Calafate, J.-C. Cano, P. Manzoni, Vacamobil: Vanet car mobility manager for omnet ++ , in: Communications Workshops (ICC), 2013 IEEE International Conference on, 2013, pp. 10571061. doi:10.1109/ICCW.2013.6649393. 
[26] Video Traces, http://trace.eas.asu.edu, acessed: January 15, 2013.

[27] P. Pinol, A. Torres, O. Lopez, M. Martinez, M. Malumbres, Evaluating hevc video delivery in vanet scenarios, in: Wireless Days (WD), 2013 IFIP, 2013, pp. 1-6. doi:10.1109/WD.2013.6686539. 